\title{
COMPARISON OF LIPID FRACTIONS OF ICTERIC SAMPLES USING THREE DEVICES
}

\author{
Ummul Khair, Asvin Nurulita, Darwati Muhadi
}

Department of Clinical Pathology, Faculty of Medicine, Hasanuddin University/Dr. Wahidin Sudirohusodo Hospital, Makassar. E-mail: oche_indicis@yahoo.co.id

\begin{abstract}
Lipid fraction assessment in the laboratory includes cholesterol, HDL, LDL, and Triglycerides. The icteric sample is characterized by elevated levels of bilirubin and dark yellow appearance of sample. This research was to compare of the lipid fractions of icteric samples by using three devices. This was a cross-sectional study at the Clinical Pathology Laboratory of the Dr. Wahidin Sudirohusodo, Makassar, which compared the lipid fractions of icteric samples using 3 (three) devices (Pentra, Biomajesty, Conelab) during April - June 2018. For the statistical analysis SPSS program was used. A total sample of 50 indicated that the cholesterol levels measured by Biomajesty were lower than Pentra 400 ( $p<0.001$ ), while the cholesterol levels measured by Conelab were significantly lower than Pentra 400 ( $p>0.001)$, but not significantly different with Biomajesty $(p<0.05)$. HDL level measured by Biomajesty did not differ significantly with Pentra 400 ( $p>0.05)$. High Density Lipoprotein (HDL) levels by Conelab were significantly lower than Pentra $400(p<0.001)$ and Biomajesty $(p<0.01)$. High-density lipoprotein levels measured by Biomajesty were the lowest compared to Pentra and Conelab. Low-Density Lipoprotein (LDL) level measured by Biomajesty did not differ significantly with Pentra $400(p>0.05)$ while LDL level measured by Conelab was significantly lower than Pentra $400(p<0.001)$ and Biomajesty $(p<0.001)$. It was shown that the range of TG measured by Biomajesty was the lowest (more accurate) than Pentra 400 and Conelab. Triglycerides (TG) levels measured by Biomajesty were significantly lower than Pentra $400(p<0.01)$, while TG levels measured by Conelab were not significantly different than Pentra $400(p>0.05)$, but significantly higher than Biomajesty $(p<0.001)$. The research showed that Biomajesty was an accurate device for the measurements of HDL, LDL, and TG levels of icteric samples, whereas Conelab was an accurate device for cholesterol measurement or icteric samples.
\end{abstract}

Key words: Lipid fraction, icteric sample, Pentra, Biomajesty, Conelab

\section{INTRODUCTION}

The icteric sample is a sample characterized by both an $2 \mathrm{mg} / \mathrm{dL}$ increase of bilirubin levels and dark yellow color. Icterus often occurs in hemolytic disease, liver disease, and bile duct obstruction. Icteric serum samples will affect other laboratory test values. For instance, the icteric serum can affect measurements at a wavelength of 400-500 nm due to the dark yellow color of the specimen causing the photometer unable to measure optical density perfectly. Lipid fractions that are commonly examined in the laboratory are cholesterol, HighDensity Lipoprotein (HDL). Low-Density Lipoprotein (LDL), and Triglycerides (TG). ${ }^{1-3}$

The interference mechanism by all clinical chemistry devices will affect the ability to react to chemicals in other reagents, causing a declining value and spectral interference during color measurement. ${ }^{4,5}$

Similarly, a research by Haughton et al. in America in 2007 showed that the levels of cholesterol and triglyceride decreased in icteric samples, without any information about the device used. Unlike the previous research, icteric samples with decreased results were commonly reported at the Dr. Wahidin Sudirohusodo General Hospital, especially in lipid fractions test. ${ }^{6}$

In fact, there are certain devices for lipid fraction and bilirubin levels measurement, namely $A B X$ Pentra 400, Biomajesty JCA-BM610/C, and Konelab-30. The performance of those three devices is based on enzymatic reactions and chemical analysis using Spectrophotometric by colorimetric principle. The three devices not only have the same enzymatic reaction, but also different interferences that will affect the results of the lipid fraction levels measurement. Enzymatic reactions which occur based on the parameters used with the test principle can be seen as follows: ${ }^{7-9}$

As a result, this research was focused on the levels of lipid fraction in icteric samples measured by three 
1. Enzymatic reactions in cholesterol test

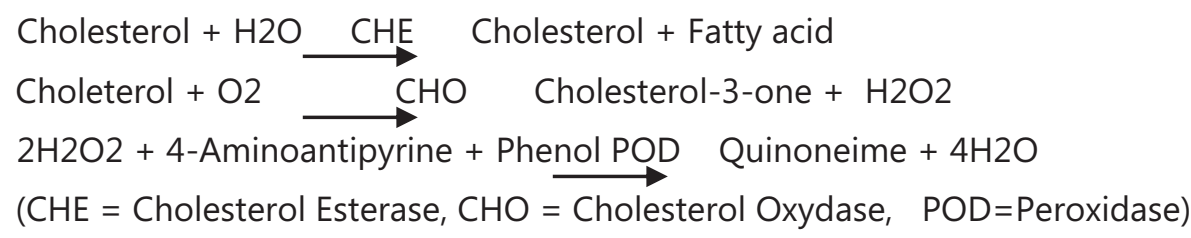

2. Enzymatic reactions in HDL test
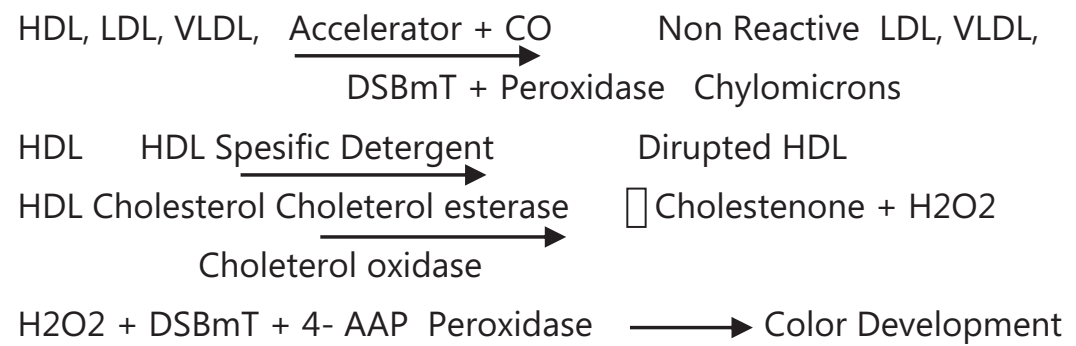

3. Enzymatic reactions in LDL test

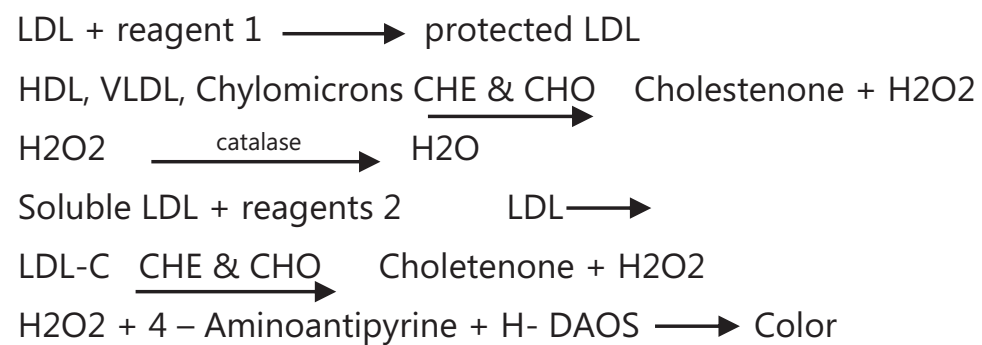

4. Enzymatic reactions in Triglyceride test

Tryglicerides LPL $\longrightarrow$ Glycerol + fatty acid

Glycerol + ATP GK $\longrightarrow$ Glycerol-3-Phosphate + ADP

Glycerol-3-Phosphate + O2 GPO Dhydroxyceton phosphate + $\mathrm{H}_{2} \mathrm{O} 2$

$2 \mathrm{H} 2 \mathrm{O} 2+$ Aminoantipyrine + 4--Chlo rophenol $\stackrel{\mathrm{POD}}{\longrightarrow}$ Quinemineine $+\mathrm{HCL}+4 \mathrm{H} 2 \mathrm{O}$

devices used in the Dr. Wahidin Sudirohusodo General Hospital, Makassar.

This research aimed to analyze the ratio of lipid fractions in icteric samples measured by all three devices (ABX Pentra 400, Biomajesty JCA-BM610/C, Konelab-30).

\section{METHODS}

This research was an analytical study using a cross-sectional design conducted at the Clinical Pathology Laboratory Installation of Dr. Wahidin Sudirohusodo General Hospital, Makassar from January to April 2018. The inclusion criteria for sampling in this research were all icteric samples (50 samples) with the total bilirubin levels of $2.26-27.81 \mathrm{mg} / \mathrm{dL}$ and were examined for lipid fractions with three devices (ABX Pentra 400, Biomajesty JCA-BM610/C and Konelab -30), enzymatic reactions, and chemical analysis using the Spectrophotometric method with colorimetric principles. ${ }^{7-10}$

Data analysis was performed using SPSS. The statistical analysis test used was Wilcoxon Signed Ranks test since all data were not normally distributed. The test results were considered to be statistically significant if the $p$-value was $<0.05 .^{10}$

Ethical clearance was obtained from the Health

Research Ethics Commission, Faculty of Medicine, Hasanuddin University/Dr. Wahidin Sudirohusodo Hospital Makassar with number: 305/H4.8.4.5.3.1/ PP36-KOMETIK/2018.

\section{RESULTS AND DISCUSSION}

This research was carried out in the Laboratory Installation of Dr. Wahidin Sudirohusodo General Hospital, Makassar during January-April 2018. Fifty icteric samples with the total bilirubin levels of $2.26-27.81 \mathrm{mg} / \mathrm{dL}$ were used to examine lipid fractions as shown in table and graph analysis.

Table 1 showed that the mean cholesterol level 
Table. 1 Comparison of lipid fraction levels

\begin{tabular}{llcccccc}
\hline Lipid Fraction (mg/dl) & Instruments & Median & Median & Mean & SD & P \\
\hline Cholesterol & PENTRA 400 & $28.2-451.6$ & 141.1 & 167.9 & 100.1 & & \\
& BIOMAJESTY & $0.9-401.0$ & 130.0 & 157.9 & 97.9 & & $0.000^{\mathrm{a}}$ \\
& KONELAB & $25.0-372.0$ & 131.5 & 148.0 & 83.8 & $0.000^{\mathrm{b}}$ & $0.086^{\mathrm{c}}$ \\
$\mathrm{HDL}$ & PENTRA 400 & $0.9-66.5$ & 21.1 & 23.1 & 19.0 & & \\
& BIOMAJESTY & $0.4-63.0$ & 11.0 & 21.1 & 18.8 & & $0.721^{\mathrm{a}}$ \\
& KONELAB & $1.0-65.0$ & 6.5 & 19.5 & 20.1 & $0.000^{\mathrm{b}}$ & $0.001^{\mathrm{c}}$ \\
$\mathrm{LDL}$ & PENTRA 400 & $7.9-293.5$ & 77.0 & 89.5 & 64.6 & & \\
& BIOMAJESTY & $17.0-193.0$ & 81.0 & 89.4 & 39.3 & & $0.158^{\mathrm{a}}$ \\
$\mathrm{T}$ & KONELAB & $0.0-183.0$ & 42.5 & 54.1 & 52.0 & $0.000^{\mathrm{b}}$ & $0.000^{\mathrm{c}}$ \\
& PENTRA 400 & $45.0-575.0$ & 139.0 & 199.0 & 139.6 & & \\
& BIOMAJESTY & $17.0-509.0$ & 119.5 & 171.2 & 126.3 & & $0.003^{\mathrm{a}}$ \\
& KONELAB & $46.0-584.0$ & 144.5 & 201.3 & 140.9 & $0.142^{\mathrm{b}}$ & $0.000^{\mathrm{c}}$ \\
\hline
\end{tabular}

${ }^{a}$ BIOSMAJESTY vs. PENTRA $400{ }^{b}$ KONELAB vs. PENTRA $400 \quad{ }^{c}$ KONELAB vs. BIOMAJESTY

measured by Pentra 400 analyzer, Biomajesty analyzer, and Konlabe analyzer were $167.9 \mathrm{mg} / \mathrm{dL}$ (28.2-451.6) $\quad 157.9 \mathrm{mg} / \mathrm{dL}(0.9-0.41)$, and 148.0 $\mathrm{mg} / \mathrm{dL}$ (25.0-372.0), respectively, suggesting that the mean cholesterol level measured by Biomajesty analyzer was lower than that measured by Pentra 400 analyzer ( $p<0.001$ ). It also indicated that the mean cholesterol level measured by Konelab analyzer was significantly lower than that measured by Pentra 400 analyzer ( $p<0.001$ ), but not significantly different from that measured by Biomajesty analyzer $(p>0.05)$.

Moreover, Table 1 also showed that the mean HDL level measured by Pentra 400 analyzer, Bomajesty analyzer, and Konlabe analyzer were $23.1 \mathrm{mg} / \mathrm{dL}(0.9-66.5), 21.1 \mathrm{mg} / \mathrm{dL}$ (0.4-63.0), and $19.5 \mathrm{mg} / \mathrm{dL}$ (1.0-65.0), respectively. This indicated that the mean HDL level measured by Biomajesty analyzer was not significantly different from that measured by Pentra 400 analyzer ( $p>0.05)$. It also indicated that the mean HDL level measured by Konelab analyzer was significantly lower than that measured by Pentra 400 analyzer $(p<0.001)$ and Biomajesty analyzer $(p<0.01)$.

Furthermore, the Table 1 also showed that the mean LDL level measured by Pentra 400 analyzer, Biomajesty analyzer, and Konelab analyzer were 89.5 mg/dL (7.9-293.5), 89.4 mg/dL (17.0-193.0), and $54.1 \mathrm{mg} / \mathrm{dL}$ (0.0-183.0), respectively. This indicated that the mean LDL level measured by Biomajesty analyzer was not significantly different from that measured by Pentra 400 analyzer ( $p>0.05)$. It also indicated that the mean LDL level measured by Konelab analyzer was significantly lower than that measured by Pentra 400 analyzer $(p<0.001)$ and Biomajesty analyzer $(p<0.001)$.
Table 1 also showed that the mean triglyceride level measured by Pentra analyzer, Biomajesty analyzer, and Konaleb analyzer were 199.0 mg/dL (45.0-575.0), $171.2 \mathrm{mg} / \mathrm{dL}$ (17.0-509.0), and $201.3 \mathrm{mg} / \mathrm{dL}$ (46.0-584.0), respectively. This indicated that the mean triglyceride level measured by Biomajesty analyzer was significantly lower than that measured by Pentra 400 analyzer ( $p<0.01$ ). It also indicated that the mean triglyceride level measured by Konelab was not significantly different from that measured by Pentra 400 analyzer ( $p>0.05$ ), but significantly higher than that measured by Biomajesty analyzer $(p<0.001)$.

Based on the results of this research using 50 icteric samples with the total bilirubin levels of 2.26 - $27.81 \mathrm{mg} / \mathrm{dL}$, there was no significant difference in levels of lipid fraction consisting of $\mathrm{HDL}$, LDL, TG measurement using Biomajesty and Pentra devices ( $p>0.05)$. The range of the three parameters measured by Biomajesty and Pentra devices was the lowest one (more appropriate) compared to that in Pentra and Konelab devices. From thecholesterol levels measurement, the results of Konelab analyzer was significantly lower ( $p<0.001$ ) than those of Pentra analyzer, but not significantly different from the those of Biomajesty devices ( $p>0.05)$. In other words, the results of Konelab analyzer were not as extreme as those of Pentra and Biomajesty devices.

Cholesterol is derived from food and regulated by both endogenous and exogenous synthesis in the body. Cholesterol in food will be metabolized into the intestine and hydrolyzed by cholesterol esterase derived from the pancreas. Free cholesterol formed will be subsequently absorbed by the intestinal mucosa with chylomicrons as a transport molecule to the lymphatic system, and finally moved to the 
venous circulation. Approximately $70 \%$ of cholesterol is esterified, while $30 \%$ is free form. Since lipids are insoluble in water, they require a 'carrier' to enter the blood circulation. The carrier is a protein called lipoprotein. Circulating lipoproteins consist of particles of various sizes which also contain cholesterol, triglycerides, and proteins in different amounts that enable each lipoprotein to have different densities .

The largest to lowest density lipoproteins consecutively are chylomycrons, Very Low-Density Lipoprotein (VLDL), Low-Density Lipoprotein (LDL), Intermediate-Density Lipoprotein (IDL), and High-Density Lipoprotein (HDL). Plasma cholesterol is contained in LDL. Meanwhile, a small percentage (15-25\%) of cholesterol is contained in HDL. The exogenous pathway or fat-transporting food involves the absorption of triglycerides and cholesterol through the intestine.

In this research, after analyzed using the three devices, there was decrease of the HDL and LDL levels in icteric samples since HDL and LDL levels were synthesized in the liver. Besides, the total bilirubin level of $>6 \mathrm{mg} / \mathrm{dL}$ in this research would trigger a decrease in the results of HDL and LDL levels in all three devices. During the measurement of total bilirubin levels, the interference value of Pentra analyzer, Biomajesty analyzer, and Konelab analyzer were $30 \mathrm{mg} / \mathrm{dL}, 60 \mathrm{mg} / \mathrm{dL}$, and > $12 \mathrm{mg} / \mathrm{dL}$, respectively. It meant that the interference ability of each instrument to icteric samples was indeed different. Hence, the results of lipid fractions levels also varied. Similarly, a research by Haughton et al. in America in 2007 showed that there were decreased cholesterol and triglyceride levels in icteric samples. ${ }^{10}$

This research used spectrophotometer, a measurement using light, to calculate the absorption of light due to the interaction between light rays with a certain wavelength passed on the dye solution as the sample. Enzymes were also added during the measurement to determine the activity, such as the speed of the enzyme to convert the substrate with the UV test, which had poor ability to penetrate UV light with a certain wavelength like icteric samples. Therefore, the more icteric the sample, , the lower the results would be. It meant that there was inverse correlation between light absorption and transmission. Hence, the higher the absorption was, the lower the value of received light transmission would be. In a spectrophotometry-based measurement, a wavelength with maximum absorption was used to allow maximum absorption of light ray by the solution and the further capture by the detector.

\section{CONCLUSION AND SUGGESTION}

It was concluded that among the three devices used in the Laboratory Installation of the Dr. Wahidin Sudirohusodo General Hospital Makassar, the use of Biomajesty JCA-BM610/C analyzer was highly recommended to measure the levels of $H D L$, $L D L$, and TG in icteric samples. Furthermore, Konelab-30 analyzer was also highly recommended to measure cholesterol levels among three devices. Nevertheless, further researchwas suggested to examine each parameter using those three devices more than once or at least three times for each device.

\section{REFERENCES}

1. Stryer L. Metabolisme lipid dalam biokimia. Vol 3, Ed 5., Jakarta, Penerbit Buku Kedokteran EGC, 2014; 15-25.

2. Wohlgemuth R. Lipid metabolism. In: Biofilesonline sigma life science. Vol 5. USA, Sigma-aldrich, 2013; 185-189.

3. Frances K. Tinjauan klinis atas hasil pemeriksaan laboratorium. Vol 4, Ed 7., Jakarta, Penerbit Buku Kedokteran EGC, 2015; 57-61.

4. Bachorik PS. Collection of blood samples for lipoprotein analysis. In: Laboratory procedure manual. Indianapolis, Formerly Bochringer Mannheim, 2014; 161-176.

5. Cleeman IJ. Recommendation on lipoprotein measurement from the working group on lipoprotein measurement recommendations. In: National Cholesterol Education Program. Bethesda, 2013; 95-3044.

6. National Library of Medicine National Institutes of Health. Icteric human samples.Available from: https: //www.ncbi.nlm.nih.gov/pubmed/28397988 (accessed at 1 January, 2018)

7. Biomajesty $囚$ Jea_BM 6010_CG Diasiys Diagnostic System Gmbh, 2013.

8. Pentra 400 Clinical Chemistry Analyzer, Horiba medical, 2014.

9. Conelab Prime 30 Chemistry Analizer, Enseval Medika Prima, 2013.

10. Palmer-Peck O. Effects of hyperlipidemia on plasma sosium, potassium, and chloride measurement by indirect ion-selective electrode. In: Clinical chemistry. American Association for Clinical Chemistry. 2018; 64: 155-156. 